\title{
Optimization of shape parameter and exponent of generalized multiquadrics using genetic algorithm for static analysis of laminated composite plates
}

\author{
Song Xiang, Wei-ping Zhao, Chun Lu \\ Liaoning Key Laboratory of General Aviation \\ Shenyang Aerospace University \\ Shenyang, China \\ xs74342@sina.com, 2535693329@qq.com, 2372178@qq.com
}

\begin{abstract}
In the present paper, static analysis of laminated composite plates is studied by the meshless global collocation method based on generalized multiquadrics radial basis function. The generalized multiquadric radial basis function has the shape parameter and exponent which have the important effect in the accuracy of the approximation. The genetic algorithm is used to optimize the shape parameter and exponent of generalized multiquadric radial basis function. The deflection and stress of simply laminated composite plates under sinusoidal load are calculated using the generalized multiquadrics with optimal shape parameter and exponent. Present results of deflection and stress are compared with the analytical solutions of other literature which verify the validity and accuracy of present method.
\end{abstract}

Keywords-Static analysis; Laminated composite plates; Meshless; Radial basis function; Genetic algorithm

\section{INTRODUCTION}

The main advantage of laminated composite plates is high strength-to-weight ratios which offer a significant potential for reducing the weight of aerospace structural components. Many scholars have studied the static analysis of laminated composite plates [1-9].

Meshless global radial basis functions collocation method approximates the solution of the partial differential equations using radial basis functions and all nodes in the problem domain. The procedure of meshless collocation method for discretizing the governing equations is simple and straightforward. No mesh and numerical integration are required in this method. Ferreira et al. [2] used the multiquadrics radial basis functions and higher order theories to study the static analysis of laminated composite beams and plates. Ferreira [3] investigated the static characteristics of composite plates by thin-plate splines radial basis function. Ferreira et al. [4] analyzed the composite plates by trigonometric shear deformation theory and multiquadrics radial basis fucntion. Ferreira [6] analyzed the composite plates using a layerwise deformation theory and multiquadrics radial basis function. Ferreira et al. [7] analyzed the composite plates using higher-order shear deformation theory and a finite point formulation based on the multiquadric radial basis function. Xiang et al. [8] studied the static analysis of isotropic, sandwich and laminated plates by a meshless method based on the inverse multiquadrics radial basis function.

Genetic algorithm is a kind of search algorithm based on the mechanics of natural selection and natural genetics. While randomized, genetic algorithm is no simple random walk. It exploits historical information to speculate on new search points.

In the present paper, the genetic algorithm is utilized to optimize the shape parameter and exponent of generalized multiquadric radial basis function. The deflection and stress of simply laminated composite plates under sinusoidal load are calculated using the generalized multiquadrics with optimal shape parameter and exponent. Present results of deflection and stress are compared with the analytical solutions of other literature. The main purpose of present paper is to explore the potential of genetic algorithm in optimizing the shape parameter and exponent of generalized multiquadrics.

\section{GOVERNING EQUATIONS BASED ON THE HIGHER-ORDER SHEAR DEFORMATION THEORY}

The displacement field of higher-order shear deformation theory is as follows:.

$$
\begin{aligned}
& U=u(x, y)-z \frac{\partial w(x, y)}{\partial x}+f(z) \phi_{x}(x, y) \\
& V=v(x, y)-z \frac{\partial w(x, y)}{\partial y}+f(z) \phi_{y}(x, y) \\
& W=w(x, y)
\end{aligned}
$$

where $u, v, w, \phi_{x}$ and $\phi_{y}$ are the displacement functions. $f(z)$ is the shear stress function.

The project is sponsored by "Liaoning BaiQianWan talents Program (No.2014921048) 


$$
f(z)=\frac{h}{\pi} \sin \left(\frac{\pi z}{h}\right)
$$

The governing equations in terms of displacement are as follows:

$$
\begin{aligned}
& A_{11} \frac{\partial^{2} u}{\partial x^{2}}+A_{12} \frac{\partial^{2} v}{\partial x \partial y}-B_{11} \frac{\partial^{3} w}{\partial x^{3}}-B_{12} \frac{\partial^{3} w}{\partial x \partial y^{2}} \\
& +E_{11} \frac{\partial^{2} \phi_{x}}{\partial x^{2}}+E_{12} \frac{\partial^{2} \phi_{y}}{\partial x \partial y}+A_{66}\left(\frac{\partial^{2} u}{\partial y^{2}}+\frac{\partial^{2} v}{\partial x \partial y}\right) \\
& -2 B_{66} \frac{\partial^{3} w}{\partial x \partial y^{2}}+E_{66}\left(\frac{\partial^{2} \phi_{x}}{\partial y^{2}}+\frac{\partial^{2} \phi_{y}}{\partial x \partial y}\right)=0
\end{aligned}
$$

$$
\begin{aligned}
& A_{12} \frac{\partial^{2} u}{\partial x \partial y}+A_{22} \frac{\partial^{2} v}{\partial y^{2}}-B_{12} \frac{\partial^{3} w}{\partial x^{2} \partial y}-B_{22} \frac{\partial^{3} w}{\partial y^{3}} \\
& +E_{12} \frac{\partial^{2} \phi_{x}}{\partial x \partial y}+E_{22} \frac{\partial^{2} \phi_{y}}{\partial y^{2}}+A_{66}\left(\frac{\partial^{2} u}{\partial x \partial y}+\frac{\partial^{2} v}{\partial x^{2}}\right) \\
& -2 B_{66} \frac{\partial^{3} w}{\partial x^{2} \partial y}+E_{66}\left(\frac{\partial^{2} \phi_{x}}{\partial x \partial y}+\frac{\partial^{2} \phi_{y}}{\partial x^{2}}\right)=0
\end{aligned}
$$

$$
\begin{aligned}
& B_{11} \frac{\partial^{3} u}{\partial x^{3}}+B_{12} \frac{\partial^{3} v}{\partial x^{2} \partial y}-D_{11} \frac{\partial^{4} w}{\partial x^{4}}-D_{12} \frac{\partial^{4} w}{\partial x^{2} \partial y^{2}}+F_{11} \frac{\partial^{3} \phi_{x}}{\partial x^{3}} \\
& +F_{12} \frac{\partial^{3} \phi_{y}}{\partial x^{2} \partial y}+B_{12} \frac{\partial^{3} u}{\partial x \partial y^{2}}+B_{22} \frac{\partial^{3} v}{\partial y^{3}}-D_{12} \frac{\partial^{4} w}{\partial x^{2} \partial y^{2}}- \\
& D_{22} \frac{\partial^{4} w}{\partial y^{4}}+F_{12} \frac{\partial^{3} \phi_{x}}{\partial x \partial y^{2}}+F_{22} \frac{\partial^{3} \phi_{y}}{\partial y^{3}}+ \\
& 2 B_{66}\left(\frac{\partial^{3} u}{\partial x \partial y^{2}}+\frac{\partial^{3} v}{\partial x^{2} \partial y}\right)-4 D_{66} \frac{\partial^{4} w}{\partial x^{2} \partial y^{2}} \\
& +2 F_{66}\left(\frac{\partial^{3} \phi_{x}}{\partial x \partial y^{2}}+\frac{\partial^{3} \phi_{y}}{\partial x^{2} \partial y}\right)=q \\
& E_{11} \frac{\partial^{2} u}{\partial x^{2}}+E_{12} \frac{\partial^{2} v}{\partial x \partial y}-F_{11} \frac{\partial^{3} w}{\partial x^{3}}-F_{12} \frac{\partial^{3} w}{\partial x \partial y^{2}} \\
& +H_{11} \frac{\partial^{2} \phi_{x}}{\partial x^{2}}+H_{12} \frac{\partial^{2} \phi_{y}}{\partial x \partial y}+E_{66}\left(\frac{\partial^{2} u}{\partial y^{2}}+\frac{\partial^{2} v}{\partial x \partial y}\right) \\
& -2 F_{66} \frac{\partial^{3} w}{\partial x \partial y^{2}}+H_{66}\left(\frac{\partial^{2} \phi_{x}}{\partial y^{2}}+\frac{\partial^{2} \phi_{y}}{\partial x \partial y}\right)-A_{55} \phi_{x}=0
\end{aligned}
$$

$$
\begin{aligned}
& E_{12} \frac{\partial^{2} u}{\partial x \partial y}+E_{22} \frac{\partial^{2} v}{\partial y^{2}}-F_{12} \frac{\partial^{3} w}{\partial x^{2} \partial y}-F_{22} \frac{\partial^{3} w}{\partial y^{3}} \\
& +H_{12} \frac{\partial^{2} \phi_{x}}{\partial x \partial y}+H_{22} \frac{\partial^{2} \phi_{y}}{\partial y^{2}}+E_{66}\left(\frac{\partial^{2} u}{\partial x \partial y}+\frac{\partial^{2} v}{\partial x^{2}}\right) \\
& -2 F_{66} \frac{\partial^{3} w}{\partial x^{2} \partial y}+H_{66}\left(\frac{\partial^{2} \phi_{x}}{\partial x \partial y}+\frac{\partial^{2} \phi_{y}}{\partial x^{2}}\right)-A_{44} \phi_{y}=0
\end{aligned}
$$

III. SOLUTION OF THE GOVERNING EQUATIONS AND BOUNDARY CONDITIONS BY THE GENERALIZED MULTIQUADRIC RADIAL

\section{BASIS FUNCTION}

According to the meshless method based on the generalized multiquadric radial basis function, $u, v, w, \phi_{x}$ and $\phi_{y}$ can be approximated as follows:

$$
\begin{aligned}
& u=\sum_{j=1}^{N} \alpha_{j}^{u} g_{j}, v=\sum_{j=1}^{N} \alpha_{j}^{v} g_{j} w=\sum_{j=1}^{N} \alpha_{j}^{w} g_{j}, \\
& \phi_{x}=\sum_{j=1}^{N} \alpha_{j}^{\phi_{x}} g_{j}, \phi_{y}=\sum_{j=1}^{N} \alpha_{j}^{\phi_{y}} g_{j}
\end{aligned}
$$

where $N$ is the total node number, $\alpha_{j}^{u}, \alpha_{j}^{v}, \alpha_{j}^{w}, \alpha_{j}^{\phi_{x}}$ and $\alpha_{j}^{\phi_{y}}$ are unknown coefficients, $g_{j}$ is radial basis function. In present paper, radial basis function is the generalized multiquadric as follows:

$$
g_{j}=\left(\left(x-x_{j}\right)^{2}+\left(y-y_{j}\right)^{2}+c^{2}\right)^{\beta}
$$

The governing equations can be discretized by substituting the Eq. (8) into Eqs. (3-7) and boundary condition. The discretized governing equations and boundary conditions can be expressed as

$$
\left[\begin{array}{l}
L g \\
B g
\end{array}\right]\{\alpha\}=\left[\begin{array}{l}
q \\
0
\end{array}\right]
$$

Then

$$
\alpha=\left[\begin{array}{l}
L g \\
B g
\end{array}\right]^{-1}\left[\begin{array}{l}
q \\
0
\end{array}\right]
$$

where $L$ and $B$ represent the differential operators. Once the $\alpha$ is obtained, the Eq. (8) can be used to calculate the displacements.

\section{OPTIMIZATION OF SHAPE PARAMETER AND EXPONENT USING GENETIC ALGORITHM}

The mechanics of genetic algorithm are simple, involving copying strings and swapping partial strings. The genetic algorithm is composed of three operators: Reproduction, Crossover and Mutation. 
Reproduction is a process in which individual strings are copied according to their objection function values (fitness values). Copying strings according to their fitness values means that strings with a higher value have a higher probability of contributing the strings in the next generation.Crossover can proceed by swapping partial characters of two strings. Firstly, an integer $k$ is selected randomly $(1<k<\mathrm{L}-1, \mathrm{~L}$ is the length of string). Two new strings are created by swapping all characters between position $k+1$ and $\mathrm{L}$. Mutation is the occasional random alteration of value of a string. When the binary coding is used, this means changing a 1 to a 0 . In the present paper, optimization variables are shape parameter $\alpha_{c}$ and exponent $\beta$. The range of $\alpha_{c}$ is from 0.0001 to 5 . The range of $\beta$ is from -1 to 1 . The number of generation is 50 . The number of individual is 10. The binary coding is used. Each string has 10 characters. Each individual has 2 strings.

\section{NUMERICAL EXAMPLES}

A simply supported square laminated composite plate with side length $a$ and thickness $h(0 / 90 / 90 / 0)$ subjected to sinusoidal load $q$ is used to verify the validity of present method.

$q=q_{0} \sin \left(\frac{\pi x}{a}\right) \sin \left(\frac{\pi y}{a}\right)$

The material properties are as follows:

$E_{1}=25 E_{2} ; G_{12}=G_{13}=0.5 E_{2} ;$

$G_{23}=0.2 E_{2} ; v_{12}=0.25 ; E_{2}=1$;

In this paper, the node distribution is $13 \times 13$. Through the optimization of shape parameter and exponent, when $a / h \leq 20$, optimal shape parameter $\alpha_{c}=1.46$, optimal exponent $\beta=0.88$. When $a / h \geq 20$, optimal shape parameter $\alpha_{c}=1.97$, optimal exponent $\beta=-0.98$.

The non-dimensional maximum deflection and stress of a simply supported square laminated plate [0/90/90/0] calculated with optimal shape parameter and exponent are listed in Table 1, and compared with the exact solution of Pagano [9]. According to the Table 1, the present results are in good agreement with the results of Pagano [9].

TABLE I. MAXIMUM DEFLECTION AND STRESS OF A SIMPLY SUPPORTED SQUARE CROSS-PLY [0/90/90/0] LAMINATED PLATE UNDER SINUSOIDAL LOADS

\begin{tabular}{|c|c|c|c|c|c|}
\hline$a / h$ & Method & $\bar{w}$ & $\bar{\sigma}_{x}$ & $\bar{\sigma}_{y}$ & $\bar{\tau}_{x y}$ \\
\hline 4 & Pagano [9] & 1.954 & 0.720 & 0.666 & 0.0467 \\
\hline & Present & 1.8771 & 0.6900 & 0.6269 & 0.0401 \\
\hline 10 & Pagano [9] & 0.743 & 0.559 & 0.403 & 0.0276 \\
\hline \multirow{2}{*}{20} & Present & 0.7157 & 0.5507 & 0.3900 & 0.0253 \\
\hline & Pagano [9] & 0.517 & 0.543 & 0.309 & 0.0230 \\
\hline 100 & Present & 0.5056 & 0.5407 & 0.3045 & 0.0217 \\
\hline & Present & 0.4318 & 0.5377 & 0.2704 & 0.0201 \\
\hline
\end{tabular}

Figures 1-3 show the distributions of nondimensionalized normal stresses and shear stresses predicted by the present method through the thickness of a square laminate plate [0/90/90/0] under sinusoidal load $(\mathrm{a} / \mathrm{h}=10)$.

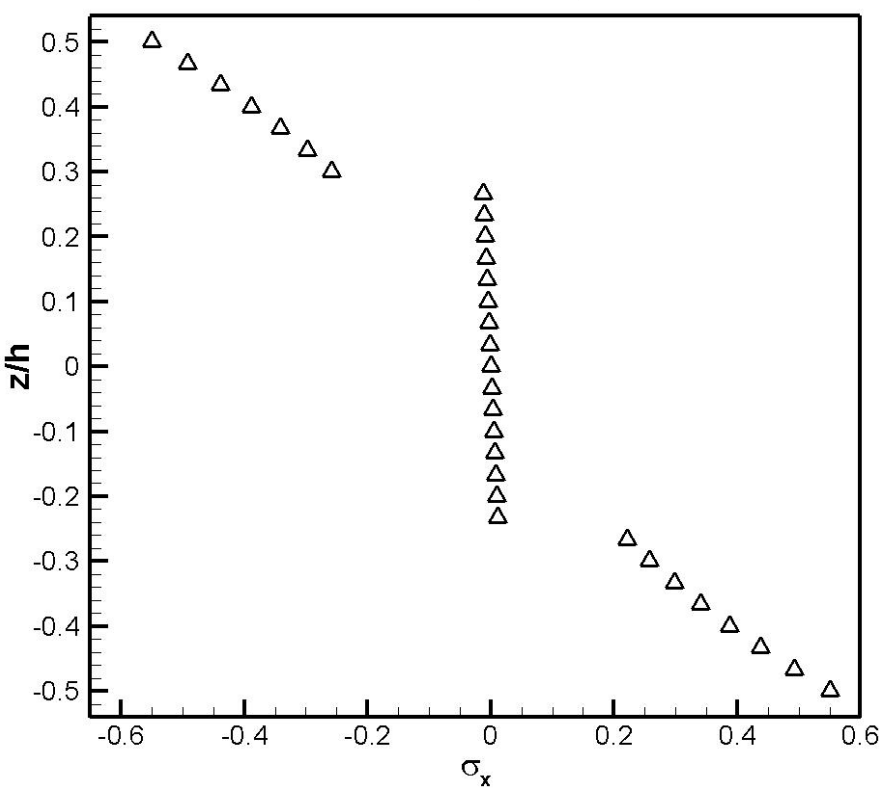

Fig. 1 Non-dimensional stress $\bar{\sigma}_{x}$ through the thickness of a square laminate plate $[0 / 90 / 90 / 0]$ under sinusoidal load $(a / h=10)$

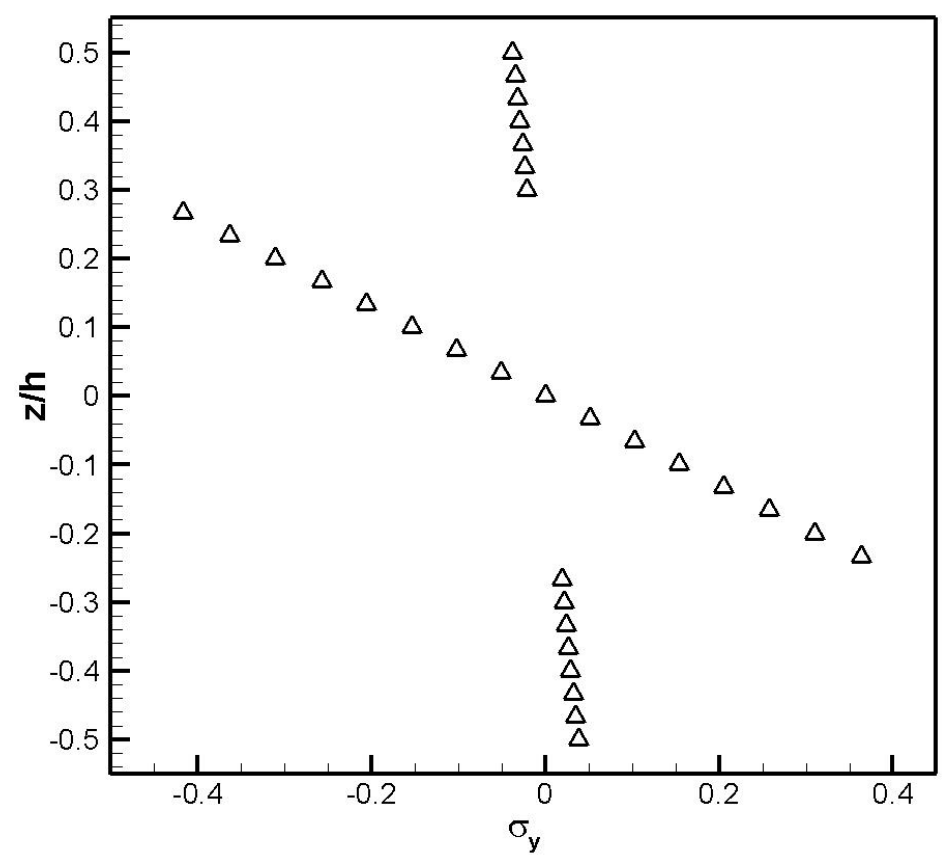

Fig. 2 Non-dimensional stress $\bar{\sigma}_{y}$ through the thickness of a square laminate plate [0/90/90/0] under sinusoidal load $(a / h=10)$ 


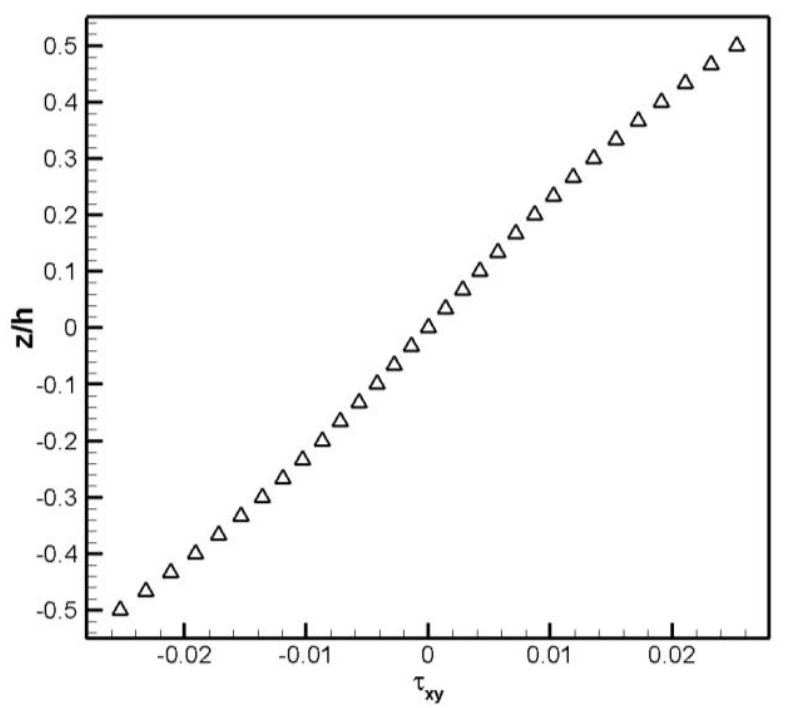

Fig. 3 Non-dimensional stress $\bar{\tau}_{x y}$ through the thickness of a square laminate plate $[0 / 90 / 90 / 0]$

\section{CONCLUSIONS}

In the present paper, static analysis of laminated composite plates is studied by the meshless global collocation method based on generalized multiquadrics radial basis function. The genetic algorithm is utilized to optimize the shape parameter and exponent of generalized multiquadric radial basis function. Through the optimization of shape parameter and exponent, when $a / h \leq 20$, optimal shape parameter $\alpha_{c}=1.46$, optimal exponent $\beta=0.88$. When $a / h \geq 20$, optimal shape parameter $\alpha_{c}=1.97$, optimal exponent $\beta=-0.98$. The deflection and stress of simply laminated composite plates under sinusoidal load are calculated using the generalized multiquadrics with optimal shape parameter and exponent. Present results of deflection and stress are compared with the analytical solutions of other literature. The numerical results show that genetic algorithm has the great potential in optimizing the shape parameter and exponent of generalized multiquadrics.

\section{References}

[1] Aydogdu M. A new shear deformation theory for laminated composite plates. Compos. Struct. 2009; 89: 94-101.

[2] Ferreira AJM, Roque CMC, Martins PALS. Radial basis functions and higher order theories in the analysis of laminated composite beams and plates. Compos. Struct. 2004; 66: 287-293.

[3] Ferreira AJM. Polyharmonic (thin-plate) splines in the analysis of composite plates. Int. J. Mech. Sci. 2005; 46: 1549-1569.

[4] Ferreira AJM., Roque CMC, Jorge RMN. Analysis of composite plates by trigonometric shear deformation theory and multiquadrics. Comput. Struct. 2005; 83: 2225-2237.

[5] Reddy JN. Mechanics of Laminated Composite Plates and Shells : Theory and Analysis 2Nd Ed, CRC Press, Boca Raton, 2004.

[6] Ferreira AJM. Analysis of composite plates using a layerwise deformation theory and multiquadrics discretization. Mech. Adv. Mater. Struct. 2005; 12: 99-112.

[7] Ferreira AJM, Roque CMC, Martins PALS, Analysis of composite plates using higher-order shear deformation theory and a finite point formulation based on the multiquadric radial basis function method. Compos. Part. B. 2003; 34: 627-636.

[8] Xiang S, Wang KM, Ai YT, Sha YD, Shi H. Analysis of isotropic, sandwich and laminated plates by a meshless method and various shear deformation theories. Compos. Struct. 2009; 91: 31-37.

[9] Pagano NJ. Exact solutions for rectangular bidirectional composites and sandwich plates. J. Compos. Mater. 1970; 4: 20-34. 